\title{
ON THE CONNECTION BETWEEN THE ELLIPTIC EQUATIONS OF THE NAVIER-STOKES TYPE AND THE THEORY OF HARMONIC FUNCTIONALS*
}

\author{
BY \\ S. M. DESHPANDE \\ Indian Institute of Science, Bangalore 12
} \begin{abstract}
the Navier-Stokes equations. differential equations which are equivalent to the Navier-Stokes equations. tions for two-dimensional, incompressible viscous flow past a body are

$$
\begin{aligned}
u_{x}+v_{y} & =0, \\
u u_{x}+v u_{y} & =-(1 / \rho) p_{x}+\nu\left(u_{x x}+u_{y y}\right), \\
u v_{x}+v u_{y} & =-(1 / \rho) p_{y}+\nu\left(v_{x x}+v_{y y}\right),
\end{aligned}
$$
\end{abstract}

Abstract. It is established in this paper, under conditions more general than those used by Millikan, that the two-dimensional incompressible viscous flows past finite bodies cannot be equivalent to a variational problem of the Euler-Lagrange type. It has thereby been possible to obtain two harmonic functionals with a close relation to

1. Introduction. Variational formulations of physical problems are well-known for their simplicity and elegance. Mostly problems governed by linear differential or integral equations have been successfully attacked by variational methods. In this paper we explore the possibility of applying variational methods to the Navier-Stokes equations. Millikan [1] has shown that the Navier-Stokes equations cannot be equivalent (except in some exceptional cases) to a variational problem, but under conditions more restrictive than those used in this paper. We find, under more general conditions, that the structure of the Navier-Stokes equations is such that we cannot get a single functional whose extremization yields the equations; instead, we obtain a system of functional

2. Analysis for two-dimensional flows. The governing partial differential equa-

where $u$ and $v$ are the components of the velocity at $(x, y)$ in a rectangular Cartesian frame, $p$ the hydrostatic pressure, $v$ the kinematic viscosity, and the suffixes $x, y$ on $u$ or $v$ indicate partial differentiation.

We consider flows past finite bodies kept in an infinite mass of fluid having a freestream velocity $U_{\infty}$ (see Fig. 1). The boundary conditions will therefore be

$$
\begin{gathered}
u=0, v=0 \text { on } S ; \\
\text { for }|x| \rightarrow \infty \text { or }|y| \rightarrow \infty, u \rightarrow U_{\infty}, v \rightarrow 0, p \rightarrow p_{\infty} ;
\end{gathered}
$$

* Received May 22, 1971; revised version received November 1, 1971. 


\section{Domain D}
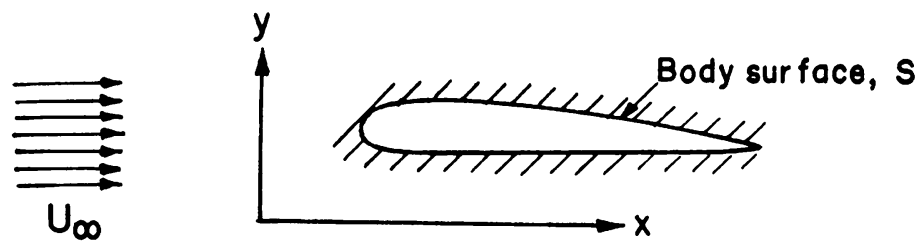

FIg. 1.

where $p_{\infty}$ is the free-stream hydrostatic pressure. We now pose the following problem: does there exist a functional $A[u, v, p]$ depending on the functions $u(x, y), v(x, y)$ and $p(x, y)$ such that its functional derivatives $\delta A / \delta p, \delta A / \delta u$ and $\delta A / \delta v$ when equated to zero yield Eqs. (2.1), (2.2)? Here $A$ is a class of functionals which we call the EulerLagrange class, defined by

$$
A[u, v, p]=\iint_{D} L\left(u, v, p, u_{x}, v_{x}, p_{x}, u_{v}, v_{v}, p_{\nu}, x, y\right) d x d y
$$

where $L$ is a function (of the variables listed in parentheses in Eq. (2.5)) satisfying certain mathematical conditions regarding continuity, differentiability, integrability, and $D$ is the domain indicated in Fig. 1. (Millikan [1] took the integrand $L$ in Eq. (2.5) to be independent of $p, p_{x}$ and $p_{y}$ and further assumed that $L$ can be expanded in a Taylor series in all the variables.)

Giving variations $\delta u, \delta v$ and $\delta p$ to $u, v$ and $p$ respectively we get, from Eq. (2.5),

$$
\begin{aligned}
\delta A= & \iint_{D}\left[\frac{\partial L}{\partial u} \delta u+\frac{\partial L}{\partial v} \delta v+\frac{\partial L}{\partial p} \delta p+\frac{\partial L}{\partial u_{x}} \delta u_{x}\right. \\
& \left.+\frac{\partial L}{\partial v_{x}} \delta v_{x}+\frac{\partial L}{\partial p_{x}} \delta p_{x}+\frac{\partial L}{\partial u_{y}} \delta u_{\nu}+\frac{\partial L}{\partial v_{\nu}} \delta v_{\nu}+\frac{\partial L}{\partial p_{y}} \delta p_{y}\right] d x d y
\end{aligned}
$$

Integrating by parts the relevant terms in Eq. (2.6), and grouping the terms involving $\delta u, \delta v$ and $\delta p$ separately, we get

$$
\begin{aligned}
& \frac{\delta A}{\delta p}=\frac{\partial L}{\partial p}-\frac{\partial}{\partial x} \frac{\partial L}{\partial p_{x}}-\frac{\partial}{\partial y} \frac{\partial L}{\partial p_{y}} \\
& \frac{\delta A}{\delta u}=\frac{\partial L}{\partial u}-\frac{\partial}{\partial x} \frac{\partial L}{\partial u_{x}}-\frac{\partial}{\partial y} \frac{\partial L}{\partial u_{\nu}} \\
& \frac{\delta A}{\delta v}=\frac{\partial L}{\partial v}-\frac{\partial}{\partial x} \frac{\partial L}{\partial v_{x}}-\frac{\partial}{\partial y} \frac{\partial L}{\partial v_{y}}
\end{aligned}
$$

provided that

$$
\left(\frac{\partial L}{\partial p_{x}}\right)_{s}=\left(\frac{\partial L}{\partial p_{y}}\right)_{s}=0 .
$$

Keeping in mind that $L$ is a function of $u, v, p, \cdots$ we get from Eqs. (2.7). (2.8) and (2.9) 


$$
\begin{aligned}
\frac{\delta A}{\delta p}= & \frac{\partial L}{\partial p}-\frac{\partial^{2} L}{\partial u \partial p_{x}} u_{x}-\frac{\partial^{2} L}{\partial v \partial p_{x}} v_{x}-\frac{\partial^{2} L}{\partial p \partial p_{x}} p_{x}-\frac{\partial^{2} L}{\partial u \partial p_{y}} u_{y}-\frac{\partial^{2} L}{\partial v \partial p_{y}} v_{y}-\frac{\partial^{2} L}{\partial p \partial p_{y}} p_{y} \\
& -\left(\frac{\partial^{2} L}{\partial u_{y} \partial p_{x}}+\frac{\partial^{2} L}{\partial u_{x} \partial p_{y}}\right) u_{x y}-\left(\frac{\partial^{2} L}{\partial v_{y} \partial p_{x}}+\frac{\partial^{2} L}{\partial v_{x} \partial p_{y}}\right) v_{x y}-2 \frac{\partial^{2} L}{\partial p_{x} \partial p_{y}} p_{x y} \\
& -\frac{\partial^{2} L}{\partial u_{x} \partial p_{x}} u_{x x}-\frac{\partial^{2} L}{\partial v_{x} \partial p_{x}} v_{x x}-\frac{\partial^{2} L}{\partial p_{x}^{2}} p_{x x}-\frac{\partial^{2} L}{\partial p_{y} \partial u_{y}} u_{y y}-\frac{\partial^{2} L}{\partial v_{y} \partial p_{y}} v_{y y}-\frac{\partial^{2} L}{\partial p_{y}^{2}} p_{y y} \\
\frac{\delta A}{\delta u}= & \frac{\partial L}{\partial u}-\frac{\partial^{2} L}{\partial u \partial u_{x}} u_{x}-\frac{\partial^{2} L}{\partial v \partial u_{x}} v_{x}-\frac{\partial^{2} L}{\partial p \partial u_{x}} p_{x}-\frac{\partial^{2} L}{\partial u \partial u_{y}} u_{y}-\frac{\partial^{2} L}{\partial v \partial u_{y}} v_{y}-\frac{\partial^{2} L}{\partial p \partial u_{y}} p_{y} \\
& -2 \frac{\partial^{2} L}{\partial u_{x} \partial u_{y}} u_{x y}-\left(\frac{\partial^{2} L}{\partial v_{x} \partial u_{y}}+\frac{\partial^{2} L}{\partial v_{y} \partial u_{x}}\right) v_{x y}-\left(\frac{\partial^{2} L}{\partial p_{y} \partial u_{x}}+\frac{\partial^{2} L}{\partial p_{x} \partial u_{y}}\right) p_{x y}-\frac{\partial^{2} L}{\partial u_{x}^{2}} u_{x x} \\
& -\frac{\partial^{2} L}{\partial v_{x} \partial u_{x}} v_{x x}-\frac{\partial^{2} L}{\partial p_{x} \partial u_{x}} p_{x x}-\frac{\partial^{2} L}{\partial u_{y}^{2}} u_{y y}-\frac{\partial^{2} L}{\partial v_{y} \partial u_{y}} v_{y y}-\frac{\partial^{2} L}{\partial p_{y} \partial u_{y}} p_{y y} \\
\frac{\delta A}{\delta v}= & \frac{\partial L}{\partial v}-\frac{\partial^{2} L}{\partial u \partial v_{x}} u_{x}-\frac{\partial^{2} L}{\partial v \partial v_{x}} v_{x}-\frac{\partial^{2} L}{\partial p \partial v_{x}} p_{x}-\frac{\partial^{2} L}{\partial u \partial v_{y}} u_{y}-\frac{\partial^{2} L}{\partial v \partial v_{y}} v_{y}-\frac{\partial^{2} L}{\partial p \partial v_{y}} p_{y} \\
& -\left(\frac{\partial^{2} L}{\partial u_{y} \partial v_{x}}+\frac{\partial^{2} L}{\partial u_{x} \partial v_{y}}\right) u_{x y}-2 \frac{\partial^{2} L}{\partial v_{y} \partial v_{x}} v_{x y}-\left(\frac{\partial^{2} L}{\partial p_{y} \partial v_{x}}+\frac{\partial^{2} L}{\partial p_{x} \partial v_{y}}\right) p_{x y} \\
& -\frac{\partial^{2} L}{\partial u_{x} \partial v_{x}} u_{x x}-\frac{\partial^{2} L}{\partial v_{x}^{2}} v_{x x}-\frac{\partial^{2} L}{\partial p_{x} \partial v_{x}} p_{x x}-\frac{\partial^{2} L}{\partial u_{y} \partial v_{y}} u_{y y}-\frac{\partial^{2} L}{\partial v_{y}^{2}} v_{y y}-\frac{\partial^{2} L}{\partial p_{y} \partial v_{y}} p_{y y} .
\end{aligned}
$$

If $\delta A / \delta p=0, \delta A / \delta u=0$ and $\delta A / \delta v=0$ are to be equivalent to Eqs. (2.1) and (2.2) then we must have

$$
\begin{aligned}
\frac{\partial^{2} L}{\partial u_{x} \partial p_{x}} & =\frac{\partial^{2} L}{\partial v_{x} \partial p_{x}}=\frac{\partial^{2} L}{\partial u_{y} \partial p_{x}}+\frac{\partial^{2} L}{\partial u_{x} \partial p_{y}}=\frac{\partial^{2} L}{\partial v_{y} \partial p_{x}}+\frac{\partial^{2} L}{\partial v_{x} \partial p_{y}}=\frac{\partial^{2} L}{\partial p_{x}^{2}}=\frac{\partial^{2} L}{\partial p_{x} \partial p_{y}} \\
& =\frac{\partial^{2} L}{\partial p_{y} \partial u_{y}}=\frac{\partial^{2} L}{\partial p_{y} \partial v_{y}}=\frac{\partial^{2} L}{\partial p_{y}^{2}}=0, \\
\frac{\partial^{2} L}{\partial u_{x} \partial v_{x}} & =\frac{\partial^{2} L}{\partial u_{x} \partial u_{y}}=\frac{\partial^{2} L}{\partial u_{x} \partial v_{y}}+\frac{\partial^{2} L}{\partial v_{x} \partial u_{y}}=\frac{\partial^{2} L}{\partial u_{y} \partial v_{y}}=\frac{\partial^{2} L}{\partial v_{x} \partial v_{y}}=0, \quad(2.14 \mathrm{a}, \mathrm{b}, \cdots, \mathrm{i}) \\
\frac{\partial^{2} L}{\partial v_{x}^{2}} & =\frac{\partial^{2} L}{\partial v_{y}^{2}}=\frac{\partial^{2} L}{\partial u_{x}^{2}}=\frac{\partial^{2} L}{\partial u_{y}^{2}}=\nu .
\end{aligned}
$$

It may be noted that Eqs. (2.14), (2.15) and (2.16) are obtained by comparing the coefficients of the second-order derivatives $u_{x x}, u_{x y}, \cdots$ etc. in $\delta A / \delta p=0, \delta A / \delta u=0$ and $\delta A / \delta v=0$ with those in Eqs. (2.1), (2.2a) and (2.2b). At this stage we cannot make a similar comparison for first-order derivatives $u_{x}, u_{y}, \cdots$ etc. because the $\partial L / \partial p$, $\partial L / \partial u$ and $\partial L / \partial v$ terms in Eqs. (2.11), (2.12) and (2.13) respectively may contain $u_{x}, u_{y}, \cdots$ etc. and thus the coefficients of these derivatives are not known. We thus conclude that the satisfaction of Eqs. (2.14), (2.15) and (2.16) is not a sufficient condition but only a necessary one for the desired equivalence. From Eqs. (2.14e), (2.14f) and (2.14i) we get

$$
L=Z\left(u_{x}, u_{y}, v_{x}, v_{y}\right)+p_{x} Z_{5}\left(u_{x}, u_{y}, v_{x}, v_{y}\right)+p_{y} Z_{6}\left(u_{x}, u_{y}, v_{x}, v_{y}\right) .
$$


We have suppressed, for convenience in writing, the variables $u, v, p, x, y$ on which the arbitrary functions $Z, Z_{5}$ and $Z_{6}$ depend. We will follow this notation throughout this paper. Further, using Eqs. (2.14a) and (2.14b), it is clear that $Z_{5}$ is independent of $u_{x}$ and $v_{x}$. Similarly, $Z_{6}$ does not depend on $u_{y}$ and $v_{y}$, in view of Eqs. (2.14g) and (2.14h). We can therefore write

$$
L=Z\left(u_{x}, u_{y}, v_{x}, v_{y}\right)+p_{x} Z_{5}\left(u_{\nu}, v_{y}\right)+p_{y} Z_{6}\left(u_{x}, v_{x}\right) .
$$

Substituting for $L$ from Eq. (2.18) in Eqs. (2.15b) and (2.15e), we get

$$
\frac{\partial^{2} Z}{\partial u_{x} \partial u_{y}}=0, \quad \frac{\partial^{2} Z}{\partial v_{x} \partial v_{y}}=0
$$

which yields the result

$$
Z=Z_{1}\left(u_{x}, v_{y}\right)+Z_{2}\left(v_{x}, u_{y}\right)+Z_{3}\left(u_{x}, v_{x}\right)+Z_{4}\left(u_{y}, v_{y}\right) .
$$

Using Eqs. (2.18), (2.20), (2.15a) and (2.15d) we get

$$
\begin{aligned}
& \frac{\partial^{2} Z_{3}}{\partial u_{x} \partial v_{x}}+p_{y} \frac{\partial^{2} Z_{6}}{\partial u_{x} \partial v_{x}}=0, \\
& \frac{\partial^{2} Z_{4}}{\partial u_{y} \partial v_{y}}+p_{x} \frac{\partial^{2} Z_{5}}{\partial u_{y} \partial v_{y}}=0
\end{aligned}
$$

which lead to

$$
\begin{array}{ll}
\frac{\partial^{2} Z_{3}}{\partial u_{x} \partial v_{x}}=0, & \frac{\partial^{2} Z_{4}}{\partial u_{y} \partial v_{y}}=0 \\
\frac{\partial^{2} Z_{6}}{\partial u_{x} \partial v_{x}}=0, & \frac{\partial^{2} Z_{5}}{\partial u_{y} \partial v_{y}}=0 .
\end{array}
$$

Eqs. (2.22) yield

$$
\begin{aligned}
& Z_{3}\left(u_{x}, v_{x}\right)=F_{1}\left(u_{x}\right)+F_{2}\left(v_{x}\right), \\
& Z_{4}\left(u_{y}, v_{u}\right)=F_{3}\left(u_{u}\right)+F_{4}\left(v_{y}\right),
\end{aligned}
$$

where $F_{1}, F_{2}, F_{3}$ and $F_{4}$ are arbitrary functions and we can absorb the first two of them in $Z_{1}$ and $Z_{2}$ and thus can drop $Z_{3}$ term in Eq. (2.20) without loss of generality. For similar reasons, the $Z_{4}$ term in the same equation can also be omitted. In view of this reasoning and Eqs. (2.23) we can write for $L$ the expression

$$
L=Z_{1}\left(u_{x}, v_{y}\right)+Z_{2}\left(v_{x}, u_{y}\right)+p_{x}\left[G_{1}\left(u_{y}\right)+G_{2}\left(v_{y}\right)\right]+p_{y}\left[G_{3}\left(u_{x}\right)+G_{4}\left(v_{x}\right)\right],
$$

where $G_{1}, G_{2}, G_{3}$ and $G_{4}$ are arbitrary functions of $u_{y}, v_{y}, u_{x}$ and $v_{x}$ respectively.

Substituting for $L$ from Eq. (2.24) in Eqs. (2.16), we get

$$
\begin{aligned}
& \frac{\partial^{2} Z_{2}}{\partial v_{x}^{2}}+p_{y} \frac{\partial^{2} G_{4}}{\partial v_{x}^{2}}=\nu \\
& \frac{\partial^{2} Z_{1}}{\partial v_{y}^{2}}+p_{x} \frac{\partial^{2} G_{2}}{\partial v_{y}^{2}}=\nu, \\
& \frac{\partial^{2} Z_{1}}{\partial u_{x}^{2}}+p_{y} \frac{\partial^{2} G_{3}}{\partial u_{x}^{2}}=\nu \\
& \frac{\partial^{2} Z_{2}}{\partial u_{\nu}^{2}}+p_{x} \frac{\partial^{2} G_{1}^{2}}{\partial u_{y}^{2}}=\nu .
\end{aligned}
$$


These equations yield

$$
\begin{aligned}
& \frac{\partial^{2} G_{4}}{\partial v_{x}^{2}}=\frac{\partial^{2} G_{2}}{\partial v_{y}^{2}}=\frac{\partial^{2} G_{3}}{\partial u_{x}^{2}}=\frac{\partial^{2} G_{1}}{\partial u_{y}^{2}}=0 \\
& \frac{\partial^{2} Z_{2}}{\partial v_{x}^{2}}=\frac{\partial^{2} Z_{1}}{\partial v_{y}^{2}}=\frac{\partial^{2} Z_{1}}{\partial u_{x}^{2}}=\frac{\partial^{2} Z_{2}}{\partial u_{y}^{2}}=\nu
\end{aligned}
$$

solving these, we get

$$
\begin{aligned}
L= & Y+Y_{1} u_{x}+Y_{2} u_{y}+Y_{3} v_{x}+Y_{4} v_{y}+Y_{5} p_{x}+Y_{6} p_{y}+\frac{1}{2} \nu\left(u_{x}^{2}+u_{y}^{2}+v_{x}^{2}+v_{y}^{2}\right) \\
& +Y_{7} p_{x} u_{y}+Y_{8} p_{x} v_{y}+Y_{9} p_{y} u_{x}+Y_{10} p_{y} v_{x}+Y_{11} u_{x} v_{y}+Y_{12} v_{x} u_{y},
\end{aligned}
$$

where $Y, Y_{1}, Y_{2}, \cdots, Y_{12}$ are arbitrary functions of $u, v, p$ and their dependence on these variables is not exhibited in conformity with the notation adopted before. Substituting for $L$ from Eq. (2.25) in Eqs. (2.14c), (2.14d) and (2.15c) we get

$$
Y_{7}+Y_{9}=Y_{8}+Y_{10}=Y_{11}+Y_{12}=0 .
$$

It is now clear that the expression for $L$ given by Eqs. (2.25) and (2.26) satisfies all the Eqs. (2.14), (2.15) and (2.16); hence, the expressions for $\delta A / \delta p, \delta A / \delta u, \delta A / \delta v$ given by Eqs. (2.11), (2.12) and (2.13) reduce to

$$
\begin{aligned}
\frac{\delta A}{\delta p}= & \frac{\partial L}{\partial p}-\frac{\partial^{2} L}{\partial u \partial p_{x}} u_{x}-\frac{\partial^{2} L}{\partial v \partial p_{x}} v_{x}-\frac{\partial^{2} L}{\partial p \partial p_{x}} p_{x}-\frac{\partial^{2} L}{\partial u \partial p_{y}} u_{y}-\frac{\partial^{2} L}{\partial v \partial p_{y}} v_{y}-\frac{\partial^{2} L}{\partial p \partial p_{y}} p_{y} \\
\frac{\delta A}{\delta u}= & \frac{\partial L}{\partial u}-\frac{\partial^{2} L}{\partial u \partial u_{x}} u_{x}-\frac{\partial^{2} L}{\partial v \partial u_{x}} v_{x}-\frac{\partial^{2} L}{\partial p \partial u_{x}} p_{x} \\
& -\frac{\partial^{2} L}{\partial u \partial u_{y}} u_{y}-\frac{\partial^{2} L}{\partial v \partial u_{y}} v_{y}-\frac{\partial^{2} L}{\partial p \partial u_{y}} p_{y}-\nu\left(u_{x x}+u_{y y}\right) \\
\frac{\delta A}{\delta v}= & \frac{\partial L}{\partial v}-\frac{\partial^{2} L}{\partial u \partial v_{x}} u_{x}-\frac{\partial^{2} L}{\partial v \partial v_{x}} v_{x}-\frac{\partial^{2} L}{\partial p \partial v_{x}} p_{x} \\
& -\frac{\partial^{2} L}{\partial u \partial v_{y}} u_{y}-\frac{\partial^{2} L}{\partial v \partial v_{y}} v_{y}-\frac{\partial^{2} L}{\partial p \partial v_{y}} p_{y}-\nu\left(v_{x x}+v_{y y}\right)
\end{aligned}
$$

The viscous terms in Eqs. (2.2a) and (2.2b) appear in the above expressions for $\delta A / \delta u$ and $\delta A / \delta v$, as must be expected. The next task is to explore the possibility of reproducing the inertia and pressure-gradient terms in these expressions, keeping in mind that $L$ must be of the type given by Eq. (2.25). It is obvious that the terms in Eq. (2.25) containing $Y, Y_{5}, Y_{6}, Y_{7}, Y_{8}$ and $Y_{11}$ cannot reproduce the inertia terms. We choose

$$
Y_{5}=u / \rho, \quad Y_{6}=v / \rho, \quad Y_{11}=\nu, \quad Y=Y_{7}=Y_{8}=0,
$$

which means

$$
\begin{aligned}
L=Y_{1} u_{x}+Y_{2} u_{y}+Y_{3} v_{x}+Y_{4} v_{y}+(u / \rho) p_{x} & +(v / \rho) p_{y} \\
& +\frac{1}{2} \nu\left(u_{x}+v_{y}\right)^{2}+\frac{1}{2} \nu\left(v_{x}-u_{y}\right)^{2} .
\end{aligned}
$$

It may be noted here that this form for $L$ satisfies Eq. (2.10). Substituting for $L$ from Eq. (2.30) into Eqs. (2.27), (2.28) and (2.29), we get 


$$
\begin{aligned}
& \frac{\delta A}{\delta p}=\left(\frac{\partial Y_{1}}{\partial p}-\frac{1}{\rho}\right) u_{x}+\left(\frac{\partial Y_{4}}{\partial p}-\frac{1}{\rho}\right) v_{\nu}+\frac{\partial Y_{2}}{\partial p} u_{y}+\frac{\partial Y_{3}}{\partial p} v_{x} \\
& \frac{\delta A}{\delta u}=\left(\frac{\partial Y_{3}}{\partial u}-\frac{\partial Y_{1}}{\partial v}\right) v_{x}+\left(\frac{\partial Y_{4}}{\partial u}-\frac{\partial Y_{2}}{\partial v}\right) v_{y}-\frac{\partial Y_{2}}{\partial p} p_{\nu}+\left(\frac{1}{\rho}-\frac{\partial Y_{1}}{\partial p}\right) p_{x}-\nu\left(u_{x x}+u_{y y}\right) \\
& \frac{\delta A}{\delta v}=\left(\frac{\partial Y_{1}}{\partial v}-\frac{\partial Y_{3}}{\partial u}\right) u_{x}+\left(\frac{\partial Y_{2}}{\partial v}-\frac{\partial Y_{4}}{\partial u}\right) u_{y}+\left(\frac{1}{\rho}-\frac{\partial Y_{4}}{\partial p}\right) p_{\nu}-\frac{\partial Y_{3}}{\partial p} p_{x}-\nu\left(v_{x x}+v_{y y}\right)
\end{aligned}
$$

We must choose

$$
\frac{\partial Y_{1}}{\partial p}=\frac{\partial Y_{2}}{\partial p}=\frac{\partial Y_{3}}{\partial p}=\frac{\partial Y_{4}}{\partial p}=0
$$

to reproduce correctly the terms $\left(u_{x}+v_{y}\right) / \rho$ in Eq. (2.31) and the pressure-gradient terms in Eqs. (2.32) and (2.33). From the expressions for $\delta A / \delta u$ and $\delta A / \delta v$ it is obvious that no functions $Y_{1}, Y_{2}, Y_{3}$ and $Y_{4}$ exist which will reproduce the inertia terms in Eqs. (2.32) and (2.33). We therefore conclude that there does not exist a single functional $A[u, v, p]$ belonging to the Euler-Lagrange class whose functional derivatives with respect to $u$ and $v$ when equated to zero would yield the momentum equations. Eqs. (2.32) and (2.33), on the other hand, possess an interesting property by virtue of which a close connection can be established between the two-dimensional Navier-Stokes equations and analytic functionals (see the Appendix). To establish this, let

$$
D[u, v, p]=\iint_{D}\left[\frac{1}{2} \nu(\operatorname{div} \mathbf{u})^{2}+\frac{1}{2} \nu(\operatorname{curl} \mathbf{u})^{2}+\frac{1}{\rho} \mathfrak{u} \cdot \operatorname{grad} p\right] d x d y
$$

and

$$
E[u, v]=-\iint_{D}\left(Y_{1} u_{x}+Y_{2} u_{v}+Y_{3} v_{x}+Y_{4} v_{y}\right) d x d y
$$

We then get

$$
\begin{aligned}
(\delta D / \delta u) & =(1 / \rho) p_{x}-\nu\left(u_{x x}+v_{y v}\right) \\
(\delta D / \delta v) & =(1 / \rho) p_{y}-\nu\left(v_{x x}+v_{y v}\right) \\
(\delta D / \delta p) & =-(1 / \rho)\left(u_{x}+v_{y}\right)
\end{aligned}
$$

and

$$
\begin{aligned}
& (\delta E / \delta u)=-\left(u v_{x}+v v_{y}\right), \\
& (\delta E / \delta v)=u u_{x}+v v_{y},
\end{aligned}
$$

if we choose $Y_{1}, Y_{2}, Y_{3}$ and $Y_{4}$ so as to satisfy

$$
\frac{\partial Y_{3}}{\partial u}-\frac{\partial Y_{1}}{\partial v}=u, \quad \frac{\partial Y_{4}}{\partial u}-\frac{\partial Y_{2}}{\partial v}=v .
$$

Using Eqs. (2.36), (2.37) and (2.38), we can write the continuity and the momentum equations equivalently as 


$$
\begin{aligned}
& \frac{\delta D}{\delta p}=0 \\
& \frac{\delta E}{\delta u}=\frac{\delta D}{\delta v} \\
& \frac{\delta E}{\delta v}=-\frac{\delta D}{\delta u}
\end{aligned}
$$

Eqs. (2.40) and (2.41) are precisely the Cauchy-Riemann conditions for $E+i D$ to be an analytic functional of $u+i v$. In order to establish the existence of $E$ compatible with Eqs. (2.38), we observe that among infinitely many solutions of Eq. (2.38) two simple solutions are

a) $Y_{1}=Y_{4}=0, Y_{2}=-\frac{1}{2} v^{2}, Y=\frac{1}{2} u^{2}$

giving

$$
E=\iint_{D} \frac{1}{2}\left(v^{2} u_{y}-u^{2} v_{x}\right) d x d y
$$

and

b) $Y_{1}=Y_{4}=0, Y_{3}=-Y_{2}=\left(u^{2}+v^{2}\right) / 2$

giving

$$
E=\iint_{D} \frac{1}{2}\left(u^{2}+v^{2}\right)\left(u_{y}-v_{x}\right) d x d y .
$$

3. Some concluding remarks.

a) Interpretation of the functionals $D$ and $E$. We observe that when $(u, v, p)$ represents a fluid dynamical motion, the div $\mathbf{u}$ term in Eq. (2.34) vanishes and so does the term $\mathbf{u}$. grad $p$, as it gives the work done by the expansion of the incompressible fluid against pressure forces. The integrand for $D$ is then just the square of the vorticity and in such a case Lamb [3] has shown that $D$ is equal to the total dissipation of energy occurring in a unit time. The drag on the body is then equal to $\rho D / U_{\infty}$.

Following Sommerfeld [4], we can define the 'mass density' of the vortex distribution equal to the vorticity, and get the result that $E$ is the negative of the net kinetic energy due to this 'mass' distribution.

b) An approximate method of solution of flow problems. In this subsection we exploit the property of analyticity of $E+i D$ for getting an approximate method to obtain flows past finite bodies. Let us presume that

$$
\begin{aligned}
& u=\sum_{k=1}^{\infty} a_{k} \phi_{k}(x, y) \\
& v=\sum_{k=1}^{\infty} b_{k} \phi_{k}(x, y), \\
& p=\sum_{k=1}^{\infty} c_{k} \chi_{k}(x, y),
\end{aligned}
$$


where $\phi_{k}$ and $\chi_{k}, k=1,2, \cdots, \infty$, are linearly independent sets of functions. One has to be careful in the choice of these sets, as $u$ and $p$ are not $L_{2}$ functions. The functionals $D$ and $E$ can then be written as

$$
\begin{aligned}
D & =g\left(a_{1}, a_{2}, \cdots ; b_{1}, b_{2}, \cdots ; c_{1}, c_{2}, \cdots\right), \\
E & =f\left(a_{1}, a_{2}, \cdots ; b_{1}, b_{2}, \cdots\right),
\end{aligned}
$$

where $f$ and $g$ are functions of a countably infinite number of variables. In fact, $f$ and $g$ are quadratic and cubic functions respectively of these variables. We can then state that $E+i D$ being an analytic functional of $u+i v$ is equivalent to $f+i g$ being analytic (holomorphic) in $a_{k}+i b_{k}, k=1,2, \cdots \infty$. This result can be easily demonstrated by the following approach. Consider the variation in $D$ :

$$
\delta D=\sum_{k=1}^{\infty}\left(\frac{\partial g}{\partial a_{k}} \delta a_{k}+\frac{\partial g}{\partial b_{k}} \delta b_{k}+\frac{\partial g}{\partial c_{k}} \delta c_{k}\right),
$$

which is equal to

$$
\iint_{D}\left(\frac{\delta D}{\delta u} \delta u+\frac{\delta D}{\partial v} \delta v+\frac{\delta D}{\delta p} \delta p\right) d x d y .
$$

Substituting for $\delta u$ and $\delta v$ from Eqs. (3.1) and (3.2) into Eq. (3.5) we get, by comparing coefficients of $\delta a_{k}, \delta b_{k}$ in the resultant expression with those in Eq. (3.4), the expressions

$$
\begin{aligned}
\frac{\partial g}{\partial a_{k}} & =\int \frac{\delta D}{\partial u} \phi_{k}(x, y) d x d y, \\
\frac{\partial g}{\partial b_{k}} & =\int \frac{\delta D}{\delta v} \phi_{k}(x, y) d x d y .
\end{aligned}
$$

Similarly, we can show that

$$
\begin{aligned}
\frac{\partial f}{\partial a_{k}} & =\int \frac{\delta E}{\delta u} \phi_{k}(x, y) d x d y, \\
\frac{\partial f}{\partial b_{k}} & =\int \frac{\delta E}{\delta v} \phi_{k}(x, y) d x d y .
\end{aligned}
$$

The functional Cauchy-Riemann conditions (2.40) and (2.41), in view of Eqs. (3.6) through (3.9), become

$$
\begin{aligned}
& \partial f / \partial a_{k}=\partial g / \partial b_{k}, \\
& \partial f / \partial b_{k}=-\partial g / \partial a_{k},
\end{aligned}
$$

thus establishing the result that $f+i g$ is analytic in $a_{k}+i b_{k}, k=1,2, \cdots, \infty$. It may be noted that the continuity equations become

$$
\partial g / \partial c_{k}=0, \quad k=1,2, \cdots, \infty .
$$

Now Eqs. (3.10), (3.11) and (3.12) are only algebraic equations (in fact, quadratic) in $a_{k}, b_{k}$ and $c_{k}$. Solution of these equations then yields a solution to the flow problem.

c) Functional power series representation of $E+i D$. If $G[w(x, y)]$ is a cubic analytic functional, then (see the Appendix) 


$$
\begin{aligned}
G[w(x, y)]= & K_{0}+\int_{D} K_{1}(x, y) w(x, y) d x d y \\
& +\int_{D} K_{2}\left(x_{1}, y_{1} ; x_{2}, y_{2}\right) w\left(x_{1}, y_{1}\right) w\left(x_{2}, y_{2}\right) d x_{1} d y_{1} d x_{2} d y_{2} \\
& +\int_{D} K_{3}\left(x_{1}, y_{1} ; x_{2}, y_{2} ; x_{3}, y_{3}\right) w\left(x_{1}, y_{1}\right) \\
& \cdot w\left(x_{2}, y_{2}\right) w\left(x_{3}, y_{3}\right) d x_{1} d y_{1} d x_{2} d y_{2} d x_{3} d y_{3}
\end{aligned}
$$

where we have denoted, for convenience in writing, multiple integration by a single integration symbol. In view of the fact that $E+i D$ is a cubic functional, analytic at that $w$ which satisfies the Navier-Stokes equations, it is clear that $E+i D$ must be equal to $G[w(x, y)]$. We may use this property for generating solutions of the NavierStokes equations. This can be achieved by assuming some specific kernels $K_{0}, K_{1}, K_{2}$ and $K_{3}$ and then by finding a $w$ which makes $E+i D$ equal to $G[w(x, y)]$. Further, if $w=0$ gives a closed curve and $w$ tends to a constant at infinity, then that $w$ represents a solution to the flow past the corresponding body.

d) Extension to three-dimensional flows. The extension of the above analysis to three dimensions is conceptually not very difficult but somewhat involved mathematically. We will not go into the analysis here but shall be content with the following observation. Following Deshpande [5] we can write the Navier-Stokes equations for three-dimensional flows as

$$
\operatorname{CURL}(\mathbf{E})=\operatorname{GRAD}(D)
$$

where CURL and GRAD are functional differential operators, $\mathbf{E}$ is a vector functional and $D$ a scalar functional defined by

$$
\begin{aligned}
E_{n} & =-\int_{V} \frac{1}{5} \epsilon_{r n t} u_{r} u_{s} D_{t s} d x_{1} d x_{2} d x_{3} \\
D & =\int_{V}\left[\frac{1}{2} \nu(\operatorname{div} \mathbf{u})^{2}+\frac{1}{2} \nu(\operatorname{curl} \mathbf{u})^{2}+\frac{1}{\rho} \mathbf{u} \cdot \operatorname{grad} p\right] d x_{1} d x_{2} d x_{3} .
\end{aligned}
$$

Here $\epsilon_{r n \ell}$ is the alternating Cartesian tensor, $u_{r}$ is the $r$ th component of the velocity vector $\mathbf{u}$ and the dummy suffix notation is used in the above equations.

Acknowledgement. The author gratefully acknowledges the help and suggestions received from his colleague Dr. A. Prabhu on the extension of the work to three-dimensional flows. The author is also grateful to the referee for many valuable suggestions.

Appendiz. The concept of an analytic functional used in this paper is closely related to the analytical functional introduced by Volterra [2]. Following Volterra we define

$$
\begin{aligned}
G[w(t)]= & K_{0}+\int_{a}^{b} K_{1}(\xi) w(\xi) d \xi+\int_{a}^{b} \int_{a}^{b} K_{2}\left(\xi_{1}, \xi_{2}\right) w\left(\xi_{1}\right) w\left(\xi_{2}\right) d \xi_{1} d \xi_{2} \\
& +\cdots+\int_{a}^{b} \cdots \int_{a}^{b} K_{n}\left(\xi_{1}, \xi_{2}, \cdots, \xi_{n}\right) w\left(\xi_{1}\right) \cdots w\left(\xi_{n}\right) d \xi_{1} \cdots d \xi_{n}+\cdots
\end{aligned}
$$

where $w(t)$ belongs to a ceratin functional field (e.g. the set of all continuous functions $w(t), t \in[a, b]), K_{n}$ is a symmetric kernel in all its variables $\xi_{1}, \xi_{2}, \cdots, \xi_{n}$ and it is 
presumed that the above series (A.1) is convergent if $|w(t)|<R$. It may be noted that the series (A.1) is a functional power series.

If we now regard $w$ as a complex function $u(x, y)+i v(x, y)$ of two variables $x$ and $y$ defined over a certain domain $D$ and further make all the kernels also into complex functions of several variables $x_{1}, y_{1} ; x_{2}, y_{2} ; \cdots$ we get

$$
\begin{aligned}
G[w(x, y)]= & K_{0}+\iint_{D} K_{1}(x, y) w(x, y) d x d y \\
& +\iiint \int_{D} K_{2}\left(x_{1}, y_{1} ; x_{2}, y_{2}\right) w\left(x_{1}, y_{1}\right) w\left(x_{2}, y_{2}\right) d x_{1} d y_{1} d x_{2} d y_{2}+\cdots ;
\end{aligned}
$$

we call this an analytic functional provided the series (A.2) converges for $|w(x, y)|<R$. We may take $u$ and $v$ as continuous functions defined on $D$ and having continuous first-order partial derivatives everywhere in $D$. We can easily show that $G[w(x, y)]$ defined by Eq. (A.2) satisfies the Cauchy-Riemann conditions

$$
\delta H / \delta u=\delta J / \delta v, \quad \delta H / \delta v=-\delta J / \delta u,
$$

where $H$ and $J$ are the real and imaginary parts respectively of $G[w(x, y)]$.

\section{ReFerences}

[1] C. B. Millikan, On the steady motion of viscous, incompressible fluids; with particular reference to a variational principle, Phil. Mag. 7, 641-662 (1929)

[2] V. Volterra, Theory of functionals and of integral and integro-differential equations, Blackie \& Son Limited, London \& Glasgow, 1931, p. 21

[3] H. Lamb, Hydrodynamics, Dover Publications, New York, 1932, p. 580

[4] A. Sommerfeld, Lectures on theoretical physics, Vol. II, Academic Press, 1964, p. 159

[5] S. M. Deshpande, Variational formulation of Navier-Stokes equations in complex function space, Department of Aeronautical Engineering, Indian Institute of Science, Bangalore, Rep. 71 FM 10, 1971 